Swarthmore College

Works

$10-8-2007$

\title{
Far-Off-Resonant Ring Trap Near The Ends Of Optical Fibers
}

\author{
Frank Moscatelli \\ Swarthmore College, fmoscat1@swarthmore.edu \\ C. Sackett \\ S. Du \\ E. Oh
}

Follow this and additional works at: https://works.swarthmore.edu/fac-physics

Part of the Physics Commons

Let us know how access to these works benefits you

\section{Recommended Citation}

Frank Moscatelli, C. Sackett, S. Du, and E. Oh. (2007). "Far-Off-Resonant Ring Trap Near The Ends Of Optical Fibers". Physical Review A. Volume 76, Issue 4. DOI: 10.1103/PhysRevA.76.043404 https://works.swarthmore.edu/fac-physics/207

This work is brought to you for free by Swarthmore College Libraries' Works. It has been accepted for inclusion in Physics \& Astronomy Faculty Works by an authorized administrator of Works. For more information, please contact myworks@swarthmore.edu. 


\title{
Far-off-resonant ring trap near the ends of optical fibers
}

\author{
Frank Moscatelli, ${ }^{1, *}$ Charles Sackett, ${ }^{2}$ Shengwang Du, ${ }^{3}$ and Eun $\mathrm{Oh}^{2,4}$ \\ ${ }^{1}$ Department of Physics and Astronomy, Swarthmore College, Swarthmore, Pennsylvania 19081, USA \\ ${ }^{2}$ Physics Department, University of Virginia, Charlottesville, Virginia 22904, USA \\ ${ }^{3}$ Edward L. Ginzton Laboratory, Stanford University, Stanford, California 94305, USA \\ ${ }^{4}$ Remote Sensing Division, U.S. Naval Research Laboratory, Washington DC 20375, USA
}

(Received 6 July 2007; published 8 October 2007)

\begin{abstract}
We propose that micrometer-sized atom traps can be created using the optical dipole force between the ends of two single-mode optical fibers carrying counterpropagating light beams of two different wavelengths from both fibers. The traps have a simple design that is feasible to implement with commercially available products. They can be used as a flexible "atom tweezer" to manipulate atoms in free space without the need for traditional focused laser beams. A particularly interesting feature is the formation of a static ring-shaped trap for properly chosen beam parameters. Furthermore, this ring can be split into two longitudinally adjacent rings. Microscopic ring traps such as this could have important applications in atom interferometry and fundamental investigations of Bose-Einstein condensates.
\end{abstract}

DOI: 10.1103/PhysRevA.76.043404

PACS number(s): 32.80.Pj, 39.25.+k, 03.75.Be

\section{INTRODUCTION}

The cooling and trapping of atoms and the attainment of Bose-Einstein condensation (BEC) [1-3] have provided numerous new tools for studying the behavior of quantum systems in different dimensions, as well as possible sources for high-precision atomic quantum sensing and information processing [4]. Most recently, ultracold atoms in twodimensional closed ring-shaped traps have attracted much research interest. They have potential applications in rotational inertial sensing, quantum computing, and studies of exotic BEC excitations [5-7]. A plethora of techniques to produce ring traps have been proposed and implemented, using magnetic [8-11] and optical [12] methods. The method proposed here is in comparison quite simple, and can be used to produce relatively small rings with radii of a few micrometers. Microscopic traps are of interest because their size is of the same order as the typical de Broglie wavelength of a Bose-Einstein condensate, making coherence effects important. Also, small rings can be expected to exhibit stronger atomic interactions. Le Kien et al. [13] have suggested that a microscopic cylindrical trap could be created using an evanescent wave surrounding a subwavelength-diameter optical fiber. However, holding cold atoms very close to a roomtemperature surface can lead to loss [14]. Our method avoids this problem while also providing true three-dimensional confinement.

We have previously reported on our work investigating macroscopic static ring trapping structures using rotating magnetic fields [15]. In the present paper we propose several simple and controllable micrometer-sized traps for ultracold rubidium atoms using a conventional single-mode optical fiber (SMF) and a two-color light field dipole potential. Our proposal does not rely on evanescent fields but is similar to traditional optical tweezers where a red-detuned Gaussian beam is focused to form a waist in free space. The basic idea

\footnotetext{
*fmoscat1@swarthmore.edu
}

is illustrated in Fig. 1. Two optical fibers are arranged end to end inside a vacuum chamber, with a small $(100 \mu \mathrm{m})$ gap in between. Each fiber carries both far-red- and far-bluedetuned light. The blue light generates a repulsive potential that is strongest near the axis of the fibers, while the red light provides an attractive potential that extends to larger radii. As Fig. 2 illustrates, the combination provides a ring-shaped trap at the midpoint between the two fibers. The ring trap parameters, including size, trap depth, and frequencies, can be easily adjusted by changing laser powers. Additionally, we will show that the ring trap can be split in the longitudinal (axial) direction [see Fig. 1(b)] so that interference between two ring BECs can be studied. A fiber-based microring trap can also be easily integrated into the advancing field of atom chips for portable and miniature-size atom-wave devices [16-18].

In Sec. II we describe the transverse and longitudinal character of the two-color optical potential and analyze the fields in order to obtain physical insight into their use as traps. In Sec. III we present results for two trapping scenarios that rely on the relative size of the mode field diameters (MFDs) for the two light colors. We numerically obtain trapping parameters such as trap depth, shapes, coherence time, and capture lifetime. Section IV examines the robustness of the traps with respect to misalignment. We present conclusions in Sec. V.

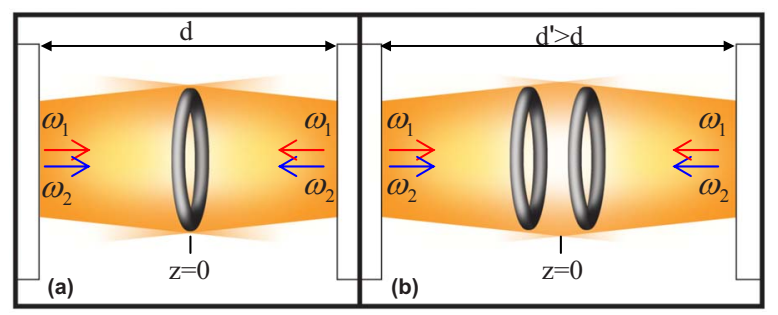

FIG. 1. (Color online) General experimental setup of fiber ring trap. (a) A single ring trap appears at the midpoint between the two fiber ends. (b) Increasing the separation causes the ring trap to split into two identical rings. 


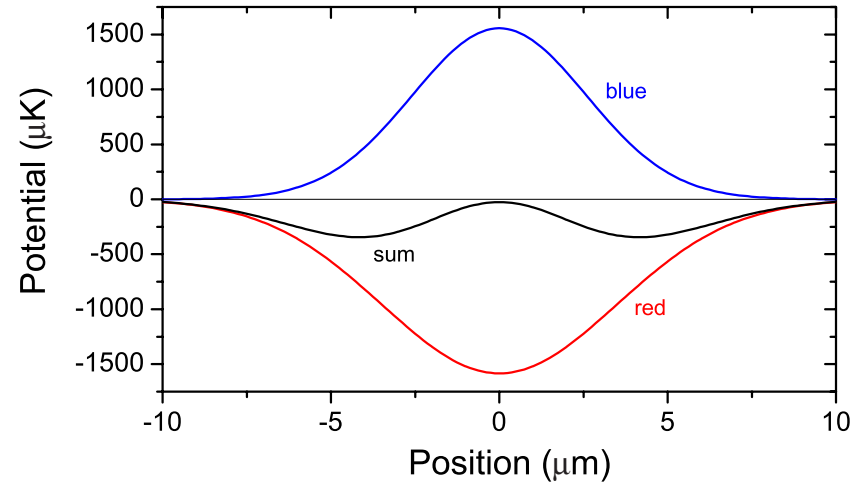

FIG. 2. (Color online) Optical potentials plotted along a line perpendicular to the fiber axes. The red-detuned light (shown in red) provides an attractive potential, while the blue-detuned light (in blue) is repulsive. The sum (black) gives a ring-shaped potential with an off-axis minimum.

\section{FIBER TRAP THEORY: MATHEMATICAL ANALYSIS}

Light exiting the output end of a SMF is Gaussian shaped, but the waist is located right at the output surface of the fiber end. Forming an atomic trap there is not possible since atoms would interact with the surface and either stick to it or become heated and thereby escape the trap. We show that the presence of an identically prepared beam from the counterpropagating direction, however, moves the minimum in the longitudinal direction away from the fiber end surface. To avoid standing waves, and hence the production of an optical lattice, the counterpropagating beam can have a slightly different frequency. This can readily be achieved using an acousto-optic modulator. The geometry is shown schematically in Fig. 1

A light field with components oscillating at $\omega_{i}$ produces a conservative optical dipole potential given by [19]

$$
U=-\frac{1}{2} \sum_{i} \alpha\left(\omega_{i}\right)\left|E\left(\omega_{i}\right)\right|^{2},
$$

where $E\left(\omega_{i}\right)$ is the electric field component and $\alpha\left(\omega_{i}\right)$ is the real part of the atomic polarizability at that frequency. This can be derived from a semiclassical Lorentz model of the atom, or from the full density matrix approach.

In alkali-metal atoms the first excited electronic configuration gives rise to two levels split by the fine-structure interval. This splitting is small compared to our proposed laser detunings, so the contribution to the total atomic polarizability from both these " $D 1$ " and " $D 2$ " transitions must be included. This yields an approximate expression for the optical potential of [20]

$$
U=\frac{3 \pi c^{2}}{2 \omega_{i}^{3}}\left(\frac{\gamma_{D 1}}{3 \Delta_{D 1}}+\frac{2 \gamma_{D 2}}{3 \Delta_{D 2}}\right) I(\rho, z)
$$

where $\gamma_{i}$ is the linewidth for transition $i, \Delta_{i}$ is the detuning from transition $i$, and $I(\rho, z)$ is the optical intensity. For a Gaussian beam carrying power $P$ in free space the intensity $I(\rho, z)$ is a function of the longitudinal distance $z$ and radial position $\rho=\left(x^{2}+y^{2}\right)^{1 / 2}$. It is given by [21]

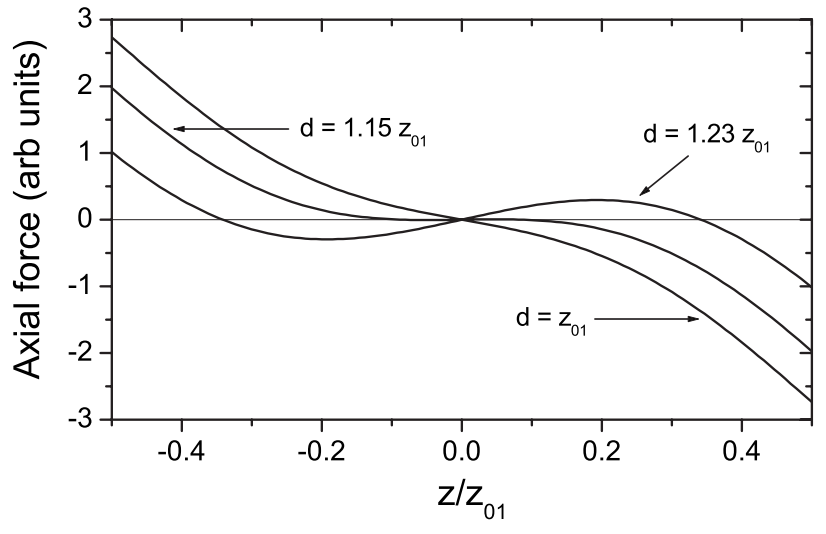

FIG. 3. Dipole force derived from Eq. (4). The equilibrium at $z=0$ is stable (i.e., a trap exists) for values of $d<1.15 z_{01}$. For values greater than this critical value, such as $d=1.23 z_{01}$, the equilibrium at $z=0$ becomes unstable but two additional stable equilibria exist. That is, the trap splits into two.

$$
I(\rho, z)=I_{0}\left(\frac{W_{0}}{W(z)}\right)^{2} \exp \left(-\frac{2 \rho^{2}}{W^{2}(z)}\right),
$$

where $z_{0}=\left(\pi W_{0}^{2}\right) / \lambda$ is the Rayleigh length, $W_{0}$ is the waist radius, $W(z)=W_{0}\left[1+\left(z / z_{0}\right)^{2}\right]^{1 / 2}$, and $I_{0}=2 P /\left(\pi W_{0}^{2}\right)$. For light exiting a SMF the position of the beam waist is at the end surface and $W_{0}$ is given by the MFD of the fiber.

To get insight into the trapping potentials and their properties, we first perform an analytical treatment for a singlefrequency trap. Many of the features for this case are relevant to the two-color scenario. For a single red-detuned field at the wavelength of $\lambda_{1}$, the global minimum of $U_{1}$ is, of course, always on the axis so we may set $\rho=0$ in Eq. (3). The on-axis potential for both fibers, one at $z=d / 2$ and the other at $z=-d / 2$, is

$$
U_{1}(\rho=0, z)=-\frac{P_{1} a_{1}}{W_{01}^{2}}\left(\frac{z_{01}^{2}}{z_{01}^{2}+(z-d / 2)^{2}}+\frac{z_{01}^{2}}{z_{01}^{2}+(z+d / 2)^{2}}\right),
$$

where $a_{1}=\left|\left(2 \alpha_{1}\right) /\left(\pi c \varepsilon_{0}\right)\right|$. Here $W_{01}$ and $z_{01}$ are, respectively, the waist radius and Rayleigh length for the red beam.

Extrema in the potential will exist wherever $d U / d z=0$. This condition yields $(z-d / 2) /\left[z_{01}^{2}+(z+d / 2)^{2}\right]^{2}$ $+(z+d / 2) /\left[z_{01}^{2}+(z-d / 2)^{2}\right]^{2}=0$. One root by inspection is $z=0$, halfway between the two fiber ends. To have a trap, of course, the extremum must be a minimum. Evaluation of the second_derivative shows that this occurs for $d<d_{\text {crit }}=2 z_{01} / \sqrt{3}$. Insight into this condition, as well as knowledge of the other roots, can be obtained by considering the following graphical argument.

Both terms in Eq. (4) are Lorentzians, one due to the fiber end located at $z=d / 2$ and the other at $z=-d / 2$. We wish to vary $d$ and investigate the roots of the first-derivative condition above. The derivatives of each term are dispersionshaped curves. Graphical analysis, as in Fig. 3, shows that, as long as the displaced curve has its maximum position below that of the minimum of the undisplaced dispersion curve, the extremum at $z=0$ will be a minimum and a trap will exist 
there. As $d$ is increased past the critical value, two other roots of the derivative condition become distinct from $z=0$ and the trap splits in two. The midpoint $z=0$ becomes a local maximum and the two split-off roots are minima.

The following can, therefore, be concluded. As the fiber end separation increases from $d=0$, a trap will exist at $z=0$. When the fiber separation reaches the critical value $d_{\text {crit }}$, the trap splits in two and each minimum moves toward the nearest fiber end. Algebraic evaluation gives the separation between the two minima in this case as

$$
\Delta z=\left[\frac{4 z_{01} W_{d 1}}{W_{01}}\left(d-\frac{z_{01} W_{d 1}}{W_{01}}\right)\right]^{1 / 2},
$$

where $W_{d 1}=W_{1}(d / 2)=W_{01}\left[1+d^{2} /\left(2 z_{01}\right)^{2}\right]^{1 / 2}$ is the value of the beam radius at the midpoint between the fiber ends. The depth of the trap is given by the value of the potential at the minimum,

$$
U_{\min }= \begin{cases}-\frac{2 a_{1} P_{1}}{W_{d 1}^{2}} & \text { for } d \leqslant d_{\text {crit }}, \\ -\frac{a_{1} P_{1}\left(2 z_{01} W_{d 1}+d W_{01}\right)}{d W_{01}^{3}} & \text { for } d>d_{\text {crit }} .\end{cases}
$$

In the case of the split trap, the barrier height between the two minima is easily evaluated as $U(0)-U_{\min }$.

The above one color analysis yields an oblate trap. A ring trap is obtained by using two different frequencies $\omega_{i}$, one tuned red of the principal atomic resonance and the other tuned blue. In general, the mode diameter of an optical fiber decreases with increasing wavelength, so if $\omega_{2}$ corresponds to the blue-detuned light, the Gaussian beam waists will satisfy $W_{01}>W_{02}$. Furthermore, shorter-wavelength light diffracts at a smaller angle, so the blue light will typically be confined more closely to the axis for all $z$. The longitudinal behavior is qualitatively identical to that of the singlefrequency trap discussed above. We therefore limit the analysis to the radial direction alone, and in particular assume that the fibers are close enough to create a single trap at $z=0$. As a function of $\rho$, the total potential is

$$
U(\rho, z=0)=-\frac{2 a_{1} P_{1}}{W_{d 1}^{2}} e^{-2 \rho^{2} / W_{d 1}^{2}}+\frac{2 a_{2} P_{2}}{W_{d 2}^{2}} e^{-2 \rho^{2} / W_{d 2}^{2}},
$$

where $P_{2}, a_{2}$, and $W_{d 2}$ are defined as above, but for the blue-detuned light.

We obtain extrema at $\rho=0$ and at values of $\rho$ that satisfy

$$
\frac{a_{1} P_{1} W_{d 2}^{4}}{a_{2} P_{2} W_{d 1}^{4}}=\exp \left[2 \rho^{2}\left(\frac{1}{W_{d 1}^{2}}-\frac{1}{W_{d 2}^{2}}\right)\right] .
$$

From the second derivative we conclude that the root at $\rho=0$ is a single minimum if $a_{1} P_{1} / W_{d 1}^{4}>a_{2} P_{2} / W_{d 2}^{4}$. Under this circumstance, however, Eq. (8) has no solution since $W_{d 1}>W_{d 2}$. This means that a single nonring trap would exist in the center between the fiber ends. This is obvious since in the limit of vanishingly small $P_{2}$ (blue light) the trap is essentially a single-color, oblate trap. As $P_{2}$ is increased, the inequality above is violated and the position $\rho=0$ becomes a local maximum. But then Eq. (8) can be solved to give a minimum at

$$
\rho_{m}=\left[\frac{W_{d 1}^{2} W_{d 2}^{2}}{2\left(W_{d 1}^{2}-W_{d 2}^{2}\right)} \ln \left(\frac{a_{2} P_{2} W_{d 1}^{4}}{a_{1} P_{1} W_{d 2}^{4}}\right)\right]^{1 / 2},
$$

thus producing a ring-shaped trap. In the limit of very large $P_{2}$, however, $\rho_{m} \rightarrow \infty$. This is also reasonable considering that a single-wavelength, blue-detuned field of this kind cannot form a trap.

The critical ratio of red to blue power at which the ring trap forms is

$$
\frac{P_{1}}{P_{2}}=\frac{a_{2} W_{d 1}^{4}}{a_{1} W_{d 2}^{4}}=\frac{a_{2} W_{01}^{4}}{a_{1} W_{02}^{4}}\left(\frac{4+d^{2} / z_{01}^{2}}{4+d^{2} / z_{02}^{2}}\right)^{2} .
$$

For ratios larger than this an oblate trap exists at $\rho=0$ and for smaller values there is a ring trap of radius given by Eq. (9). The depth of the ring trap is easily calculated numerically.

\section{FIBER TRAP THEORY: NUMERICAL ANALYSIS}

Here we present numerical results showing how the trap parameters-radius, depth, etc.-vary with power and fiber separation. We consider trapping $\mathrm{Rb}$ atoms, using light at $\lambda_{1}=1064 \mathrm{~nm}$ and $\lambda_{2}=647 \mathrm{~nm}$, obtainable from an yttrium aluminum garnet laser and an Ar-Xe ion laser, respectively. The SM600 fiber from Fibercore Inc. is capable of transmitting both these wavelengths in a single mode. The specified MFD's are $W_{01}=6.45 \mu \mathrm{m}$ and $W_{02}=4.7 \mu \mathrm{m}$. We set $P_{1}=95 \mathrm{~mW}$ and $P_{2}=55 \mathrm{~mW}$.

In Fig. 4, we plot the total potential versus transverse and longitudinal position emphasizing various features. The contour plots of the optical potential in temperature units are shown as a function of the radial and longitudinal coordinates. In Fig. 4(a), the fiber separation $d$ is $100 \mu \mathrm{m}$, and a global minimum is clearly present between the two fibers located at $z= \pm d / 2$, and is in the shape of a ring of radius about $4 \mu \mathrm{m}$. In Fig. 4(b), the fiber separation is $160 \mu \mathrm{m}$ and two longitudinally separated ring minima are observed. Transverse and longitudinal plots through the global minima confirm this and indicate the depth and radius of the trap. These are shown in Figs. 5(a) and 5(b).

It is evident that the longitudinal variation through the trap minimum is more shallow than that of the transverse direction. The transverse depth is about $350 \mu \mathrm{K}$, whereas along the longitudinal direction it is about $30 \mu \mathrm{K}$. The longitudinal oscillation frequency near the minimum is $\omega_{z} \approx 2 \pi \times 100 \mathrm{~Hz}$, while in the radial direction $\omega_{r} \approx 2 \pi \times 3.5 \mathrm{kHz}$.

The lifetime of atoms in the trap will be limited by spontaneous emission. The light scattering rate in general is given by the Rayleigh formula

$$
R_{s}=\frac{1}{6 \pi \epsilon_{0}^{2} \hbar c^{4}} \sum_{i} \omega_{i}^{3} \alpha^{2}\left(\omega_{i}\right) I\left(\omega_{i}\right) .
$$

Again taking only the contributions of the $D 1$ and $D 2$ lines, the total scattering rate for both beams is calculated to be $1.2 \mathrm{~s}^{-1}$, giving a coherence time of $830 \mathrm{~ms}$ and a heating rate of $0.21 \mu \mathrm{K} / \mathrm{s}$.

It should be noted that at a distance of nearly $50 \mu \mathrm{m}$ from the fiber end the van der Waals, or Casimir-Polder, interac- 

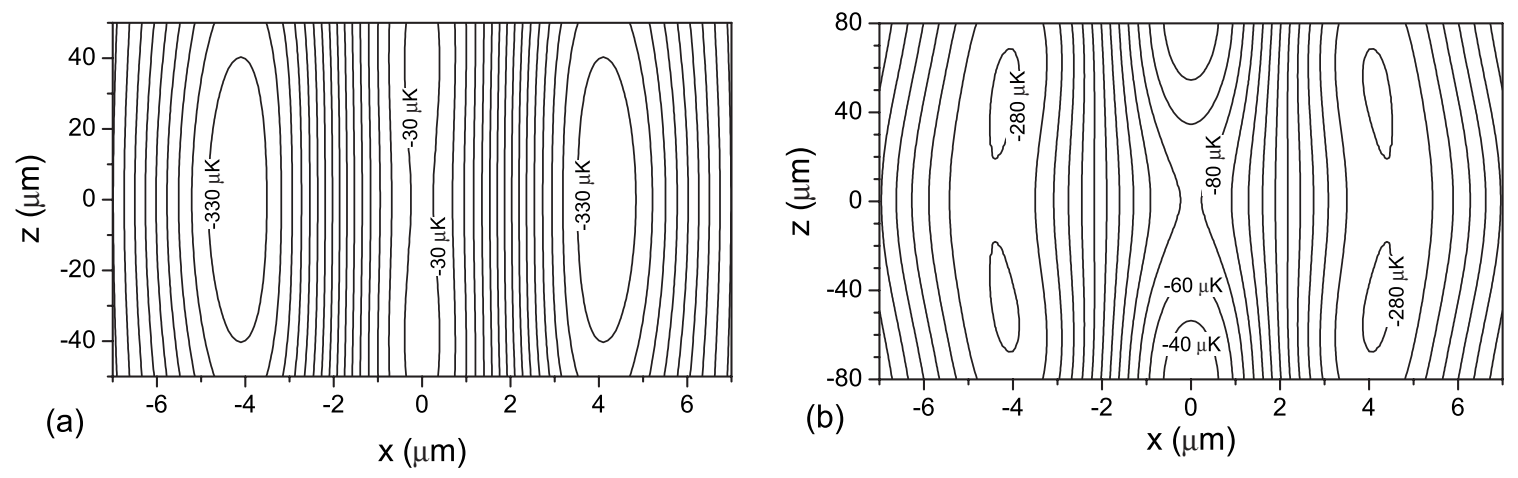

FIG. 4. Contour plot of potential as a function of $z$ and $x$ resulting in a ring trap for $d=(\mathrm{a}) 100$ and (b) $160 \mu \mathrm{m}$. Each contour is $20 \mu \mathrm{K}$.

tion between the atoms and the surface material is totally negligible. This is not the case with the other microtraps which rely on evanescent light surrounding a rectangular waveguide etched on monolithic chips [22,23].

The trap can be loaded by moving a BEC into it using any number of techniques including waveguide technologies $[24,25]$. The relatively shallow longitudinal depth of the trap can be used to further evaporatively cool the BEC. This is through contact with the fiber end surfaces. Such surface evaporative cooling has been successfully used in BEC formation on chips [14].

Figure 6 shows separation of two the traps from the center as a function of fiber separation distance $d$.
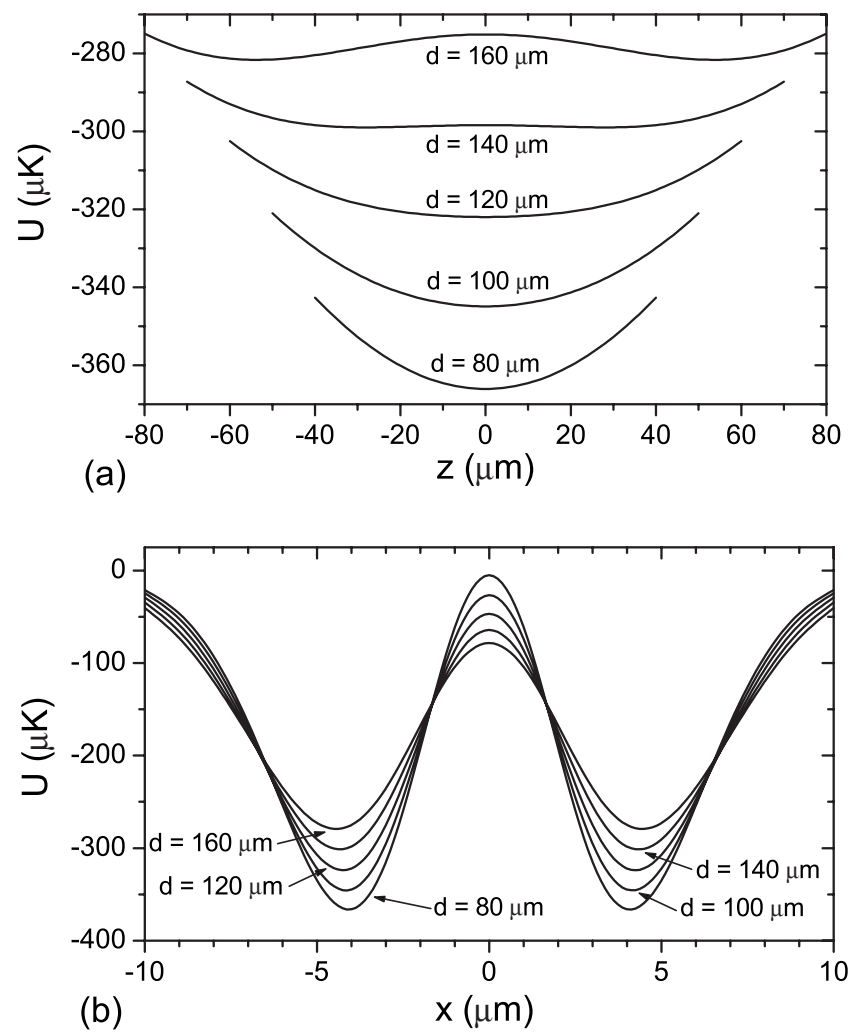

FIG. 5. Longitudinal (a) and transverse (b) plots of the potential of the ring trap for various $d$. In each case, the profile is taken through the actual location of the trap minimum.

\section{TRAP ROBUSTNESS}

Finally, we investigate the robustness of this trapping scheme with respect to misalignments of the two fibers. Traps relying on evanescent fields in channel waveguide $[22,23]$ suffer from degradation in performance due to imperfections in the guide manufacturing. This effect is exacerbated since the traps in that scheme are very close to the waveguide surface. Burke et al. [23] show that scattering of even $0.1 \%$ of one of the colors due to these imperfections cuts the maximum trapping depth by $50 \%$. In the scenario we propose, the traps are far from surfaces and quite unaffected by such concerns.

On the other hand, the numerical analysis we presented in Sec. II assumed that the two fibers were perfectly collinear. We can investigate the effects of lateral displacement of the two fibers by introducing a misalignment $\delta$ into Eq. (3).

Figure 7 is a two-dimensional contour plot of the potential under the misalignment effect of a lateral displacement $\delta=1 \mu \mathrm{m}$. At this displacement the overall quality of the trap is intact. The ring is simply "canted" by $15^{\circ}$. Above $2 \mu \mathrm{m}$ displacement, the trap begins to suffer serious degradation and leakage. Alignment to within $1 \mu \mathrm{m}$ could be accomplished by launching light into one fiber and positioning the other fiber to maximize transmission. With MFD's on the order of $4 \mu \mathrm{m}$, single-micrometer sensitivity with respect to alignment is quite feasible. This is a much less stringent

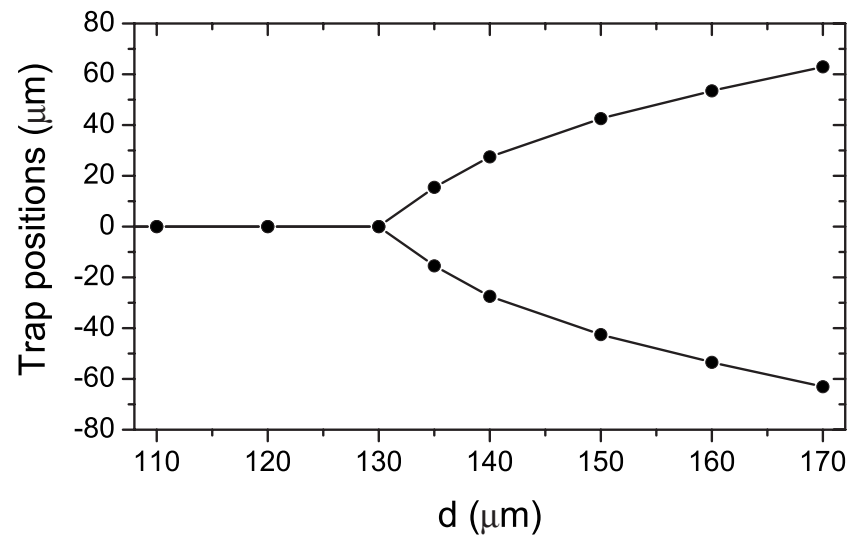

FIG. 6. Distance of the trap minimum from the center $(z=0)$ as a function of $d$ for the ring trap. 


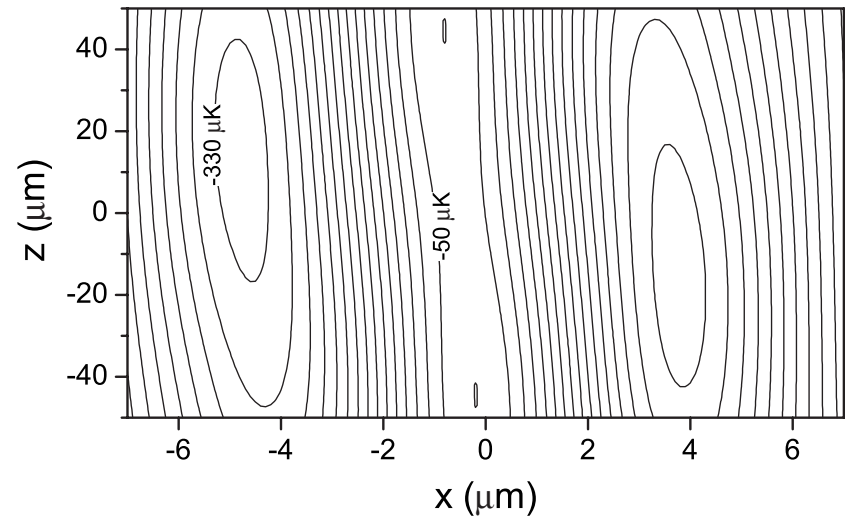

FIG. 7. Contour plot of the potential in the $x-z$ plane with one fiber displaced by $1 \mu \mathrm{m}$. Each contour is $20 \mu \mathrm{K}$.

requirement than with the channel waveguide traps mentioned earlier. Their surfaces must be free of imperfections to a fraction of the optical wavelength.

\section{CONCLUSIONS}

We propose a technique for producing a ring-shaped microtrap at a comfortable distance from the end of a flexible optical fiber. Moreover, the trap can be longitudinally split into two separate rings. The ring configuration is a highly sought-after trap shape for ultracold atoms since it can be used to realize highly sensitive inertial sensors such as atom gyroscopes and atom interferometers. The positional flexibility of the trap allows for atomic manipulation necessary in experiments involving quantum entanglement, quantum coherence, and BEC transport, for example.

It is to be emphasized that the parameters we selected for this trap-the two wavelengths, the optical powers at these wavelengths, and the fiber-are all commercially available. In the future, we plan to closely follow developments in high-index fibers made from novel materials that allow a larger range of wavelength difference in single-mode propagation. Such flexibility would make it possible to vary the ring size and depth in situ.

\section{ACKNOWLEDGMENTS}

We would like to thank the reviewer for excellent and insightful comments and recommendations. We are grateful for fruitful conversations with Paul Lett of NIST, Gaithersburg, MD. F.M. was supported in this work by Swarthmore College and NIST. C.S. was supported by NSF and DARPA. S.D. was supported by the DARPA, the U.S. Air Force Office of Scientific Research, and the U.S. Army Research Office. E.O. was supported by the Office of Naval Research.
[1] E. L. Raab, M. Prentiss, A. Cable, S. Chu, and D. E. Pritchard, Phys. Rev. Lett. 59, 2631 (1987).

[2] M. H. Anderson, J. R. Ensher, M. R. Matthews, C. E. Wieman, and E. A. Cornell, Science 269, 198 (1995).

[3] K. B. Davis, M.-O. Mewes, M. R. Andrews, N. J. van Druten, D. S. Durfee, D. M. Kurn, and W. Ketterle, Phys. Rev. Lett. 75, 3969 (1995).

[4] K. G. Petrosyan and L. You, Phys. Rev. A 59, 639 (1999).

[5] T. L. Gustavson, P. Bouyer, and M. A. Kasevich, Phys. Rev. Lett. 78, 2046 (1997).

[6] J. V. Porto, S. Rolston, B. Laburthe Tolra, C. J. Williams, and W. D. Phillips, Philos. Trans. R. Soc. London, Ser. A 361, 1417 (2003).

[7] P. Engels, I. Coddington, P. C. Haljan, V. Schweikhard, and E. A. Cornell, Phys. Rev. Lett. 90, 170405 (2003).

[8] N. H. Decker, C. S. Lee, J. H. Thywissen, S. P. Smith, M. Drndic, R. M. Westervelt, and M. Prentiss, Phys. Rev. Lett. 84, 1124 (2000).

[9] M. B. Crookston, P. M. Baker, and M. P. Robinson, J. Phys. B 38, 3289 (2005).

[10] J. A. Sauer, M. D. Barrett, and M. S. Chapman, Phys. Rev. Lett. 87, 270401 (2001).

[11] J. Schmiedmayer, Phys. Rev. A 52, R13 (1995).

[12] B. Depret, P. Verkerk, and D. Hennequin, J. Phys. IV 12, 137 (2002).
[13] F. Le Kien, V. I. Balykin, and K. Hakuta, Phys. Rev. A 70, 063403 (2004).

[14] D. M. Harber, J. M. McGuirk, J. M. Obrecht, and E. A. Cornell, J. Low Temp. Phys. 133, 229 (2003).

[15] J. Wu, F. Moscatelli, and E. Oh, Opt. Express 14, 4969 (2006).

[16] W. Hansel, P. Hommelhoff, T. W. Hansch, and J. Reichel, Nature (London) 413, 498 (2001).

[17] H. Ott, J. Fortagh, G. Schlotterbeck, A. Grossmann, and C. Zimmermann, Phys. Rev. Lett. 87, 230401 (2001).

[18] S. Du, M. B. Squires, Y. Imai, L. Czaia, R. A. Saravanan, V. Bright, J. Reichel, T. W. Hansch, and D. Z. Anderson, Phys. Rev. A 70, 053606 (2004).

[19] See, for example, J. D. Jackson, Classical Electrodynamics, 3rd ed. (Wiley, New York, 1999).

[20] C. R. Grimm, M. Weidemuller, and Y. B. Ovchinnikov, Adv. At., Mol., Opt. Phys. 42, 95 (2000).

[21] See, for example B. E. A. Saleh and M. Teich, Fundamentals of Photonics, 2nd ed. (Wiley, New York, 2007).

[22] A. H. Barnett, S. P. Smith, M. Olshanii, K. S. Johnson, A. W. Adams, and M. Prentiss, Phys. Rev. A 61, 023608 (2000).

[23] J. P. Burke, Jr., S. T. Chu, G. W. Bryant, C. J. Williams, and P. S. Julienne, Phys. Rev. A 65, 043411 (2002).

[24] L. Plaja and L. Santos, Phys. Rev. A 65, 035602 (2002).

[25] K. Bongs, S. Burger, S. Dettmer, D. Hellweg, J. Arlt, W. Ertmer, and K. Sengstock, Phys. Rev. A 63, 031602(R) (2001). 